\title{
Ring-Polymer Quantization of Photon Field in Polariton Chemistry
}

\author{
Sutirtha N. Chowdhury, Arkajit Mandal, and Pengfei Huo* \\ Department of Chemistry, University of Rochester, 120 Trustee Road, Rochester, New York \\ 14627, United States \\ E-mail: pengfei.huo@rochester.edu
}

\section{Abstract}

We use the ring-polymer (RP) representation to quantize the radiation field inside an optical cavity to investigate polariton quantum dynamics. Using a charge transfer model coupled to an optical cavity, we demonstrate that the RP quantization of the photon field provides accurate rate constants of the polariton mediated electron transfer (PMET) reaction compared to the Fermi's Golden rule. Because RP quantization uses extended phase space to describe the photon field, it significantly reduces the computational costs compared to the commonly used Fock states description of the radiation field. Compared to the other quasi-classical descriptions of the photon field, such as the classical Wigner model, the RP representation provides a much more accurate description of the polaritonic quantum dynamics, because it properly preserves the quantum distribution of the photonic DOF throughout the quantum dynamics propagation of the molecule-cavity hybrid system, whereas the classical Wigner model fails to do so. This work demonstrates the possibility of using the ring-polymer description to treat the quantized radiation field in polariton chemistry, offering an accurate and efficient approach for future investigations in cavity quantum electrodynamics.

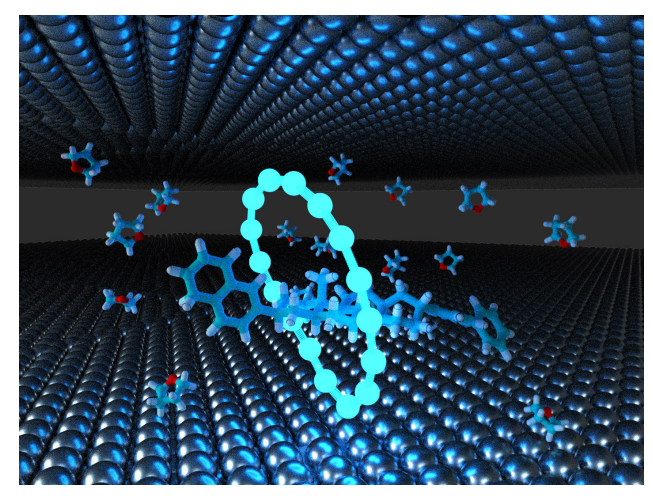

Introduction. Coupling molecules to the quantized radiation field inside an optical cavity creates a set of new photon-matter hybrid states, so-called polaritons. These light-matter hybrid polaritons have shown a great promise to control chemical reactivities $^{1-7}$ in a general way by tuning the fundamental properties of photons and provides a new paradigm for enabling chemical transformations that can profoundly impact catalysis, energy production, and the field of chemistry at large. Theoretical investigations have played a crucial role in unraveling the fundamental principles of polariton chemistry. ${ }^{4-20}$ Despite encouraging progress, accurately and efficiently simulating these polariton quantum dynamics processes opens a brand new challenge in theoretical chemistry.

In previous works of polariton chemistry, the cavity photon field has been treated quantum mechanically through Fock states, ${ }^{8,9,12,13,15,18,19}$ grid points, ${ }^{4,11,21}$ coherent states, ${ }^{7}$ and Polarized Fock states. $^{22}$ These approaches provide an accurate description of the quantum light-matter interactions. They are, however, computationally demanding as they involve full quantum description of the radiation modes and are often limited in terms of how many modes that can be explicitly quantized.

The similarity (or even the isomorphism) between the vibrational quantization of nuclei in molecules and the photonic quantization of the radiation mode inside the cavity has inspired the quasi-classical description of the photon field. In fact, quasi-classical quantization (in the actionangle quasi-classical description) of the photon field has been historically used to treat molecule-laser field interaction by Miller and co-workers. ${ }^{23,24}$ Recent example of the quasi-classical description of the radiation mode in cavity QED includes the classical Wigner model ${ }^{25-27}$ as well as the symmetric quasi-classical window approach. ${ }^{27,28}$ These 
quasi-classical approaches can significantly reduce the computational cost due to the quasi-classical treatment of the field. However, the classical Wigner model ${ }^{25,26}$ is not expected to preserve the quantum distribution associated with the photon field, ${ }^{28}$ which often leads to the incorrect quantum dynamics due to the leakage of the zero-point energy (ZPE). ${ }^{29-31}$

These shortcomings of the quasi-classical treatment can be readily addressed with the recently developed state-dependent ring polymer molecular dynamics (RPMD) approaches. ${ }^{32-37}$ These approaches are based upon the imaginary-time pathintegral description of the quantum DOF in the extended phase space. ${ }^{38-43}$ The classical evolution in RPMD preserves its initial quantum distribution captured by the ring-polymer Hamiltonian, and it is free of the zero-point energy leaking problem. ${ }^{29,43}$ With the recent development of statedependent RPMD approaches, ${ }^{32-37}$ one can accurately capture both non-adiabatic electronic transitions in molecular systems while explicitly quantizing either the nuclear DOF or even the photonic mode through the ring polymer description.

In this work, we quantize the photon field with the ring-polymer description and simulate the polariton mediated electron transfer reaction through the non-adiabatic RPMD (NRPMD) approach. ${ }^{31,32,37}$ We demonstrate that the ringpolymer quantization of the photon field provides an accurate polaritonic quantum dynamics of the molecule-cavity hybrid system, compared to the quasi-classical description from the classical Wigner model. We further provide an interesting interpretation of the influence from the cavity field on the molecule as a fluctuating (Peierls type) coupling that facilitate the charge transfer.

Theoretical Approach. We start with the Pauli-Fierz (PF) non-relativistic QED Hamiltonian $^{13,20,21,44,45}$ to describe the molecular system $\hat{H}_{\mathrm{m}}$ coupled to the radiation field $\hat{H}_{\mathrm{p}}=\left(\hat{a}^{\dagger} \hat{a}+\right.$ $\left.\frac{1}{2}\right) \hbar \omega_{\mathrm{c}}$ inside an optical cavity, under the long wavelength limit. ${ }^{20}$ The PF Hamiltonian is

$$
\begin{aligned}
& \hat{H}_{\mathrm{PF}}=\hat{H}_{\mathrm{m}}+\left(\hat{a}^{\dagger} \hat{a}+\frac{1}{2}\right) \hbar \omega_{\mathrm{c}}+\boldsymbol{\chi} \cdot \hat{\boldsymbol{\mu}}\left(\hat{a}^{\dagger}+\hat{a}\right)+\frac{(\boldsymbol{\chi} \cdot \hat{\boldsymbol{\mu}})^{2}}{\hbar \omega_{\mathrm{c}}} \\
& =\hat{H}_{\mathrm{m}}+\frac{1}{2} \hat{P}_{\mathrm{c}}^{2}+\frac{1}{2} \omega_{\mathrm{c}}^{2}\left(\hat{Q}_{\mathrm{c}}+\sqrt{\frac{2}{\hbar \omega_{\mathrm{c}}^{3}}} \chi \cdot \hat{\boldsymbol{\mu}}\right)^{2}
\end{aligned}
$$

where $\hat{a}^{\dagger}$ and $\hat{a}$ are the photonic creation and annihilation operator, respectively, and $\hat{Q}_{\mathrm{c}}=$ $\sqrt{\hbar / 2 \omega_{\mathrm{c}}}\left(\hat{a}^{\dagger}+\hat{a}\right)$ and $\hat{P}_{\mathrm{c}}=i \sqrt{\hbar \omega_{\mathrm{c}} / 2}\left(\hat{a}^{\dagger}-\hat{a}\right)$ are the photon field coordinate and momentum operators, with $\omega_{\mathrm{c}}$ as the photon frequency inside the cavity. Further, $\chi=\sqrt{\frac{\hbar \omega_{\mathrm{c}}}{2 \epsilon_{0} \mathcal{V}}} \hat{\mathbf{e}}$ characterizes the lightmatter interaction. The unit vector $\hat{\mathbf{e}}$ is along the field polarization direction, $\mathcal{V}$ is the quantization volume for the cavity-photon field, and $\varepsilon_{0}$ is the permittivity inside the cavity. Finally, $\hat{\boldsymbol{\mu}}$ is the total molecular dipole operator (for both electrons and nuclei). $\hat{H}_{\mathrm{PF}}$ is a pure real Hamiltonian, and the photonic DOF can be viewed as an additional "nuclear" DOF, hence computationally treated in that way.

The central idea of this letter is to quantize the photonic DOF through the ring-polymer description. ${ }^{39,40,43}$ We treat both the nuclear DOF $\mathbf{R}$ as well as the photonic DOF $Q_{\mathrm{c}}$ on an equal footing, and denote the "nuclear" DOF in the hybrid system as $\mathbf{X}=\left\{\mathbf{R}, Q_{\mathrm{c}}\right\}$, with the corresponding momenta $\boldsymbol{\Pi}=\left\{\mathbf{P}, P_{\mathrm{c}}\right\}$. For a given diabatic Hamiltonian $\hat{H}=\hat{\mathbf{T}}+\hat{V}_{0}(\hat{\mathbf{X}})+\sum_{i j} V_{i j}(\hat{\mathbf{X}})|i\rangle\langle j|$, the NRPMD approach ${ }^{32}$ suggests that there is an isomorphic Hamiltonian ${ }^{31,33,37}$ as follows

$$
\begin{aligned}
& H_{\mathcal{N}}=\sum_{\alpha=1}^{\mathcal{N}} \frac{1}{2 \mathbf{M}} \mathbf{\Pi}_{\alpha}^{2}+V_{0}\left(\mathbf{X}_{\alpha}\right)+\frac{\mathbf{M}}{2 \beta_{\mathcal{N}}^{2} \hbar^{2}}\left(\mathbf{X}_{\alpha}-\mathbf{X}_{\alpha-1}\right)^{2} \\
& +\frac{1}{2 \hbar} \sum_{i j} V_{i j}\left(\mathbf{X}_{\alpha}\right)\left(\left[\mathbf{q}_{\alpha}\right]_{i}\left[\mathbf{q}_{\alpha}\right]_{j}+\left[\mathbf{p}_{\alpha}\right]_{i}\left[\mathbf{p}_{\alpha}\right]_{j}-\delta_{i j} \hbar\right)
\end{aligned}
$$

where the coordinate $\mathbf{X}$ (with the corresponding mass $\mathbf{M}$ ) are quantized through the extended phase space description with $\mathcal{N}$ copies (the number of the imaginary time slices) $\left\{\mathbf{X}_{\alpha}\right\}$ of the original coordinates that are harmonically coupled to each other. This is commonly referred to as the ring polymer. The diabatic states $\{|i\rangle\}$ are mapped onto a set of mapping oscillators $\left\{q_{i}, p_{i}\right\}$ through the Meyer-Miller-Stock-Thoss (MMST) formalism, ${ }^{46,47}$ which are then extended to $\mathcal{N}$ copies $\left\{\left[\mathbf{q}_{\alpha}\right]_{i},\left[\mathbf{p}_{\alpha}\right]_{i}\right\}$ as well in the NRPMD Hamiltonian. ${ }^{31,33,37}$ The above Hamiltonian provides accurate non-adiabatic quantum dynamics and at the same time, explicitly captures nuclear quantum effects, as demonstrated in several model systems in previous studies. ${ }^{31,32,37}$

Fig. 1 illustrates quantizing photon field through Fock states as well as through the ring polymer description. Fig. 1A presents the quantum evolution of the light-matter hybrid system under the Fock state representation of the photon field. 
The nuclear wavepacket evolves among the photondressed electronic states $|\mathrm{D}, n\rangle=|\mathrm{D}\rangle \otimes|n\rangle$ (the donor electronic state with $n$ photons inside the cavity) and $|\mathrm{A}, m\rangle=|\mathrm{A}\rangle \otimes|m\rangle$ (the acceptor electronic state with $m$ photons inside the cavity), where $|n\rangle$ and $|m\rangle$ are the Fock states (eigenstates) of the vacuum photon field $\hat{H}_{\mathrm{p}}=\left(\hat{a}^{\dagger} \hat{a}+\frac{1}{2}\right) \hbar \omega_{\mathrm{c}}$. While Fock states provide exact quantum mechanical description of the cavity mode and polariton quantum dynamics, a lot of them are required to achieve a converged results for treating the lightmatter interactions, especially when the coupling strength is in the strong and ultra-strong coupling regime. ${ }^{21}$ In addition, the required number of Fock states will grow exponentially when multiple cavity modes are considered, ${ }^{27}$ making this approach computationally expensive.

|D)
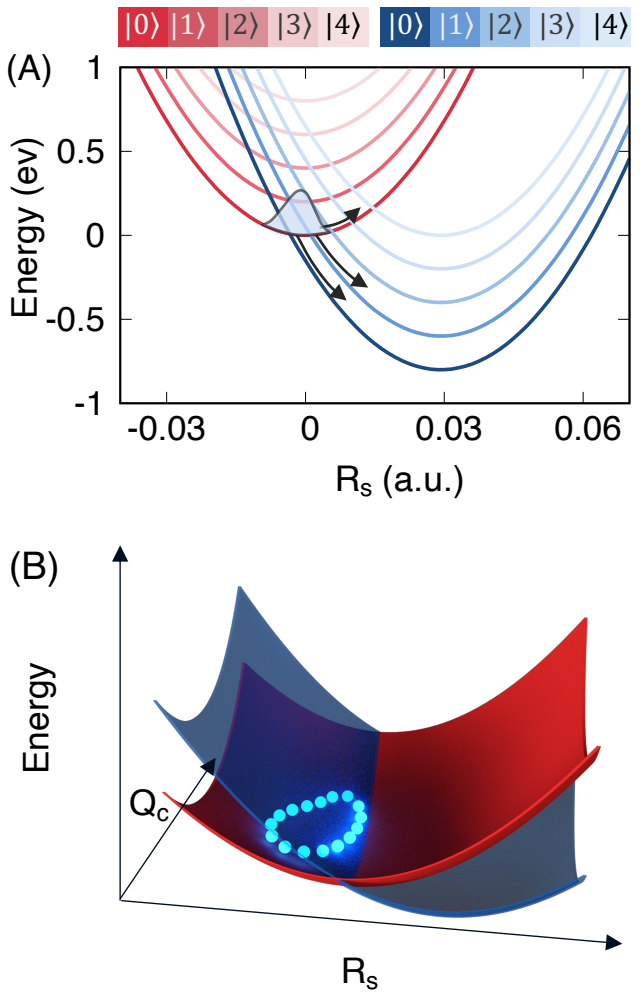

Figure 1: Schematic illustrations of the polariton mediated electron transfer model. (A) The model system described with explicit Fock state quantization of photon field coordinate $Q_{\mathrm{c}}$, where $|\mathrm{D}\rangle$ and $|\mathrm{A}\rangle$ refers the donor and acceptor electronic states, and $|n\rangle \in$ $\{|1\rangle,|2\rangle \ldots\}$ represents the vacuum's Fock states. (B) Schematic illustration of the ring polymer quantization (cyan beads) of the photonic (and nuclear) DOFs. The cavity donor (red) and the acceptor (blue) diabatic surfaces are depicted as a function of the solvent coordinate $R_{\mathrm{s}}$ and the photonic coordinate $Q_{\mathrm{c}}$.
Fig. 1B schematically presents the quantum evolution of a light-matter hybrid system when using the ring-polymer quantization. Here, the electronic DOF are described with two diabatic states, $|\mathrm{D}\rangle$ (red) and $|\mathrm{A}\rangle$ (blue). The nuclei $\mathbf{R}$ and the photon field coordinate $Q_{\mathrm{c}}$ are quantized with the ringpolymer representation. The ring-polymer evolves on the 2-dimensional diabatic electronic potential energy surfaces and undergoes non-adiabatic transitions between $|\mathrm{D}\rangle$ and $|\mathrm{A}\rangle$. Compared to the exponential scaling of the Fock state quantization, the computational cost of quantizing photon field with ring-polymer scales linearly when considering multiple radiation modes.

Model System. In this paper, we consider a donor-acceptor charge transfer model for the molecular Hamiltonian

$$
\begin{aligned}
\hat{H}_{\mathrm{m}}= & \frac{\hat{P}_{\mathrm{s}}^{2}}{2 M_{\mathrm{s}}}+\sum_{i} U_{i}|i\rangle\langle i|+V_{\mathrm{DA}}(|\mathrm{D}\rangle\langle\mathrm{A}|+| \mathrm{A}\rangle\langle\mathrm{D}|) \\
& +\sum_{i} \frac{1}{2} M_{\mathrm{s}} \omega_{\mathrm{s}}^{2}\left(R_{\mathrm{s}}-R_{i}^{0}\right)^{2}|i\rangle\langle i|+\hat{H}_{\mathrm{sb}},
\end{aligned}
$$

where $|i\rangle \in\{|\mathrm{D}\rangle,|\mathrm{A}\rangle\}$ is the diabatic donor or acceptor state, $\hat{T}_{\mathrm{s}}=\hat{P}_{\mathrm{s}}^{2} / 2 M_{\mathrm{s}}$ represents the kinetic energy operator of the solvent coordinate $R_{\mathrm{S}}$ with mass $M_{\mathrm{s}}$ and frequency $\omega_{\mathrm{s}}$. Further, $U_{i}$ is the constant diabatic energy associated with the state $|i\rangle$, with $U_{\mathrm{D}}=0$ and $U_{\mathrm{A}}=-\varepsilon$, and $V_{\mathrm{DA}}$ is the constant diabatic electronic coupling. The driving force (bias) $\Delta G$ of the reaction is $\Delta G=U_{\mathrm{A}}-U_{\mathrm{D}}=-\varepsilon$, and $\lambda=\frac{1}{2} M_{s} \omega_{s}^{2}\left(R_{\mathrm{A}}^{0}-R_{\mathrm{D}}^{0}\right)^{2}$ is the solvent reorganization energy. Further, we take $R_{\mathrm{D}}^{0}=0$ and $R_{\mathrm{A}}^{0}=\sqrt{2 \lambda / f_{0}}$, where $f_{0}$ is the force constant which is related to the solvent frequency $\omega_{\mathrm{s}}=\sqrt{f_{0} / M_{\mathrm{s}}}$. Throughout this study, we use $V_{\mathrm{DA}}=5 \mathrm{meV}$ and a solvent reorganization energy of $\lambda=650 \mathrm{meV}$. Finally, $\hat{H}_{\mathrm{sb}}=\sum_{k} \frac{P_{k}^{2}}{2 M_{k}}+\frac{M_{k} \omega_{k}^{2}}{2}\left(R_{k}-\frac{c_{k}}{M_{k} \omega_{k}^{2}} R_{\mathrm{s}}\right)^{2}$ describes the interaction between the solvent mode $R_{\mathrm{S}}$ and a dissipative bath, where $R_{k}$ represents the $k_{\text {th }}$ bath mode with a conjugate momentum $P_{k}$ and a mass $M_{k}=M_{\mathrm{s}}$. The coupling constant $c_{k}$ and the frequency $\omega_{k}$ is characterized by an ohmic spectral density $J(\omega)=\frac{\pi}{2} \sum_{k} \frac{c_{k}^{2}}{M_{k} \omega_{k}} \delta\left(\omega-\omega_{k}\right)=\eta \omega e^{-\omega / \omega_{b}}$, with a characteristic frequency $\omega_{b}$ and a friction constant $\eta$. The details of the bath discretization and all the above parameters are provided in the Supporting Information.

We further assume that the transition dipole and the permanent dipoles of the molecule are con- 
stants, which is not a function of the solvent coordinate. ${ }^{48}$ We find that within the light-matter coupling strength considered in this work, the presence of the permanent dipoles do not impact the polariton quantum dynamics, because these permanent dipoles only couple the states that are energetically off-resonance, for example, $|\mathrm{D}, n\rangle$ and $|\mathrm{D}, n \pm 1\rangle$. Hence, we completely ignore the permanent dipoles in our quantum dynamics simulations presented in the main text. In the Supporting Information, we present the results of the PMET rate obtained with the explicit permanent dipoles, which gives visually indistinguishable results from those obtained with only the transition dipole.

We further assume that the transition dipole moment $\boldsymbol{\mu}_{\mathrm{DA}}=\langle\mathrm{D}|\hat{\boldsymbol{\mu}}| \mathrm{A}\rangle$ is always aligned with the polarization direction $\hat{\mathbf{e}}$, such that

$$
\hat{\boldsymbol{\mu}} \cdot \hat{\mathbf{e}}=\boldsymbol{\mu}_{\mathrm{DA}} \cdot \hat{\mathbf{e}}(|\mathrm{D}\rangle\langle\mathrm{A}|+| \mathrm{A}\rangle\langle\mathrm{D}|) \equiv \mu_{0}(|\mathrm{D}\rangle\langle\mathrm{A}|+| \mathrm{A}\rangle\langle\mathrm{D}|),
$$

where we have defined $\mu_{0} \equiv \boldsymbol{\mu}_{\mathrm{DA}} \cdot \hat{\mathbf{e}}$.

The light-matter interaction $\hat{H}_{\text {int }}=\boldsymbol{\chi} \cdot \hat{\boldsymbol{\mu}}\left(\hat{a}^{\dagger}+\hat{a}\right)+$ $(\boldsymbol{\chi} \cdot \hat{\boldsymbol{\mu}})^{2} / \hbar \omega_{\mathrm{c}}$ in Eq. 1 for the above model system is then given as

$$
\hat{H}_{\mathrm{int}}=\hbar g_{\mathrm{c}}(|\mathrm{D}\rangle\langle\mathrm{A}|+| \mathrm{A}\rangle\langle\mathrm{D}|)\left(\hat{a}^{\dagger}+\hat{a}\right)+\frac{1}{2 \epsilon_{0} \mathcal{V}}(\hat{\boldsymbol{\mu}} \cdot \hat{\mathbf{e}})^{2},
$$

where the coupling strength $\hbar g_{\mathrm{c}} \equiv \sqrt{\frac{\hbar \omega_{\mathrm{c}}}{2 \epsilon_{0} \mathcal{V}}} \mu_{0}$, and the second term in Eq. 5 is referred to as the dipoleself energy. For a two-state system without any permanent dipole moment, $(\hat{\boldsymbol{\mu}} \cdot \hat{\mathbf{e}})^{2}=\mu_{0}^{2}(|\mathrm{D}\rangle\langle\mathrm{D}|+$ $|\mathrm{A}\rangle\langle\mathrm{A}|)$ which causes a constant energy shift for both electronic states, and hence is ignored for this special case.

Polariton Quantum Dynamics with NRPMD. We aim to compute the reduced density matrix of the light-matter hybrid system

$$
\rho_{j j}(t)=\operatorname{Tr}_{\mathbf{R}} \operatorname{Tr}_{Q_{\mathrm{c}}}\left[\hat{\rho}_{0} e^{i \hat{H} t / \hbar} \hat{\mathcal{P}}_{j} e^{-i \hat{H} t / \hbar}\right],
$$

where $\rho_{j j}(t)$ is the time-dependent population of the diabatic state $|j\rangle \in\{|\mathrm{D}\rangle,|\mathrm{A}\rangle\}, \hat{\mathcal{P}}_{j}=|j\rangle\langle j|$ is the associated projection operator, and $\operatorname{Tr}_{\mathbf{R}}$ represents the trace over all nuclear DOF (including solvent $R_{\mathrm{S}}$ and the bath $\left.\left\{R_{k}\right\}\right)$, and $\operatorname{Tr}_{Q_{c}}$ represents the trace over the photonic DOF. The initial density operator for the entire system is $\hat{\rho}_{0}=|\mathrm{D}\rangle\langle\mathrm{D}| \otimes \hat{\rho}_{\mathbf{R}} \otimes \hat{\rho}_{Q_{\mathrm{c}}}$, which is a direct product of the initial donor electronic state $|\mathrm{D}\rangle$ with the initial nuclear density operator $\hat{\rho}_{\mathbf{R}}=e^{-\beta \hat{H}_{\mathbf{R}}} / \mathcal{Z}_{\mathbf{R}}$ where $\hat{H}_{\mathbf{R}}=\frac{1}{2} M_{\mathrm{s}} \omega_{s}^{2}\left(\hat{R}_{\mathrm{s}}-R_{\mathrm{D}}^{0}\right)^{2}+\hat{H}_{\mathrm{sb}}$ and the initial distribution of the photon mode is $\hat{\rho}_{\mathrm{Q}_{\mathrm{c}}}=$ $e^{-\beta\left(\hat{a}^{\dagger} \hat{a}+\frac{1}{2}\right) \hbar \omega_{\mathrm{c}}} / \mathcal{Z}_{Q_{\mathrm{c}}}$. Further, $\mathcal{Z}_{\mathbf{R}}$ and $\mathcal{Z}_{Q_{\mathrm{c}}}$ are the corresponding partition functions for the nuclear and photonic DOFs. Choosing the initial electronic state as $|\mathrm{D}\rangle$, the distribution of the solvent coordinate $R_{\mathrm{S}}$ is centered around $R_{\mathrm{D}}^{0}$ and the distribution of the photonic coordinate is centered around $Q_{\mathrm{c}}=0$.

We use the NRPMD Hamiltonian in Eq. 2 to simulate the molecule-cavity hybrid system, and compute the time-dependent reduced density matrix $\rho_{j j}(t)$ defined in Eq. 6 through the following population expression ${ }^{31,35}$

$$
\rho_{j j}(t) \approx \int d \boldsymbol{\tau} \mathcal{P}^{0}\left(\left\{\mathbf{q}_{\alpha}, \mathbf{p}_{\alpha}\right\}\right) \rho_{\mathrm{rp}}\left(\left\{\mathbf{X}_{\alpha}, \boldsymbol{\Pi}_{\alpha}\right\}\right) \cdot \overline{\mathcal{P}}_{j}(t),
$$

where $\mathbf{X} \equiv\left\{\mathbf{R}, Q_{\mathrm{c}}\right\}, \quad \boldsymbol{\Pi} \equiv\left\{\mathbf{P}, P_{\mathrm{c}}\right\}$, and $d \boldsymbol{\tau} \equiv \int d\left\{\mathbf{X}_{\alpha}\right\} d\left\{\boldsymbol{\Pi}_{\alpha}\right\} d\left\{\mathbf{q}_{\alpha}\right\} d\left\{\mathbf{p}_{\alpha}\right\}$ with a shorthand notation $d\left\{\boldsymbol{\xi}_{\alpha}\right\}=\prod_{\alpha=1}^{\mathcal{N}} d \boldsymbol{\xi}_{\alpha}$. In addition, $\mathcal{P}^{0}\left(\left\{\mathbf{q}_{\alpha}, \mathbf{p}_{\alpha}\right\}\right)$ represents the distribution of the initial electronic variables, $\rho_{\mathrm{rp}}\left(\left\{\mathbf{X}_{\alpha}, \boldsymbol{\Pi}_{\alpha}\right\}\right)$ represents the initial ring-polymer distribution of both the nuclear and photonic DOF that corresponds to $\hat{\rho}_{\mathbf{R}} \otimes \hat{\rho}_{Q_{\mathrm{c}}}$. Finally, $\overline{\mathcal{P}}_{j}=\frac{1}{N} \sum_{\alpha} \mathcal{P}_{j}(\alpha)=$ $\frac{1}{N} \sum_{\alpha=1}^{N} \frac{1}{2}\left(\left[\mathbf{q}_{\alpha}\right]_{j}^{2}+\left[\mathbf{p}_{\alpha}\right]_{j}^{2}-1\right)$ is the electronic state estimator that has shown ${ }^{31-33,37}$ to provide accurate results for non-adiabatic dynamics.

In this work, because of the solvent $R_{\mathrm{S}}$ and the bath coordinates $\left\{R_{k}\right\}$ have low vibrational frequencies (hence exhibiting quasi-classical behavior), we use $\mathcal{N}=1$ bead for these DOFs. Thus, the initial distribution of the solvent and the bath DOFs corresponds to a pure classical distribution $\rho_{\mathbf{R}}=e^{-\beta H_{\mathbf{R}}} / \mathcal{Z}_{\mathrm{R}}$, where $H_{\mathbf{R}}=\frac{1}{2} M_{\mathrm{s}} \omega_{s}^{2}\left(R_{\mathrm{S}}-R_{D}^{0}\right)^{2}+$ $H_{\mathrm{sb}}$. For the photonic ring polymer, we treat the number of beads $\mathcal{N}$ as a convergence parameter such that the initial distribution $\rho_{\mathrm{rp}}\left(\left[Q_{\mathrm{c}}\right]_{\alpha},\left[P_{\mathrm{c}}\right]_{\alpha}\right)$ is converged. ${ }^{31,35}$ The same number of beads are used for the mapping variables, ${ }^{31}$ with the initial density $^{31,35} \mathcal{P}^{0}\left(\left\{\mathbf{q}_{\alpha}, \mathbf{p}_{\alpha}\right\}\right)=\prod_{\alpha=1}^{\mathcal{N}} \prod_{j=1} \delta\left(\mathcal{P}_{j}(\alpha)-\right.$ $\left.\rho_{j j}(0)\right)$ that is properly constrained to represent the initially occupied state $|\mathrm{D}\rangle$ through $\mathcal{P}_{j}(\alpha)=$ $\frac{1}{2}\left(\left[\mathbf{q}_{\alpha}\right]_{j}^{2}+\left[\mathbf{p}_{\alpha}\right]_{j}^{2}-1\right)=\delta_{\mathrm{D} j}$. The details of the sampling procedure are provided in the Supporting Information. All DOFs are then propagated using the Hamilton's equation of motion according to the Hamiltonian in Eq. 2. For all of the results presented in this work, a total of $10^{4}$ trajectories are used to ensure the tight convergence of popu- 
lation (based on Eq. 7), although $10^{3}$ trajectories already present the basic trend of the dynamics. With the converged population dynamics, we use a rate fitting scheme ${ }^{49,50}$ (details are provided in the Supporting Information) to obtain the PMET rate of the reaction.

Analytical Rate Expressions. For the ET model system considered in this work, the equilibrium rate constant for the non-adiabatic electron transfer reaction between donor and acceptor states can be accurately described by Marcus theory $(\mathrm{MT})^{51}$

$$
k_{\mathrm{MT}}=\frac{\left|V_{\mathrm{DA}}\right|^{2}}{\hbar} \sqrt{\frac{\pi \beta}{\lambda}} \exp \left[-\beta \frac{(\Delta G+\lambda)^{2}}{4 \lambda}\right],
$$

where $\Delta G$ is the ET driving force, $\lambda$ is the reorganization energy, $V_{\mathrm{DA}}$ is the diabatic coupling between donor and acceptor states, and $\beta=1 / k_{\mathrm{B}} T$ where $k_{\mathrm{B}}$ is the Boltzmann constant and $T$ is the temperature of the system.

For the molecule-cavity hybridized system, the polariton mediated electron transfer (PMET) occurs from a set of photon-dressed donor states $|\mathrm{D}, n\rangle$ to a set of photon dressed acceptor states $|\mathrm{A}, m\rangle$. To explicitly calculate the rates associated with each photon-dressed channel, we follow the previous theoretical work ${ }^{8,48,52}$ and use Fermi's Golden Rule (also known as the Jortner theory ${ }^{53-55}$ in ET) described as follows

$k=\sum_{n} \mathbb{P}_{n} \sum_{m} \frac{\left|F_{n m}\right|^{2}}{\hbar} \sqrt{\frac{\pi \beta}{\lambda}} \exp \left[-\beta \frac{\left(\Delta G_{n m}+\lambda\right)^{2}}{4 \lambda}\right]$,

where $F_{n m}=\left\langle\mathrm{D}, n\left|\hat{H}_{\mathrm{pl}}\right| \mathrm{A}, m\right\rangle=V_{\mathrm{DA}} \delta_{n m}+$ $\hbar g_{c}\left[\sqrt{m+1} \delta_{n, m+1}+\sqrt{m} \delta_{n, m-1}\right]$ is the effective coupling among photon dressed states, $\hat{H}_{\mathrm{pl}}=$ $\hat{H}_{\mathrm{PF}}-\hat{T}_{\mathrm{s}}-\hat{H}_{\mathrm{sb}}$ is the polariton Hamiltonian, $\hbar g_{\mathrm{c}}$ is the effective light-matter coupling (see Eq. 5), $\Delta G_{n m}=\Delta G+(m-n) \hbar \omega_{\mathrm{c}}$ is the driving force between photon-dressed states, and $\mathbb{P}_{n}=\exp \left[-\beta n \hbar \omega_{\mathrm{c}}\right] / \sum_{m} \exp \left[-\beta m \hbar \omega_{\mathrm{c}}\right]$ is the thermal population of the corresponding cavity mode. Here, we treat $n$ and $m$ as a convergence parameter and use large enough Fock states to converge the rate. Note that, the presence of zero-point energy (ZPE) of the radiation field exactly canceled inside $\mathbb{P}_{n}$, hence not directly impacting the PMET rate. The quantized nature of the photon states, on the other hand, indeed significantly influence the PMET rate through $F_{n m}$ and $\Delta G_{n m}$.
Fluctuation Mediated Rate Theory. We further view the cavity radiation mode as a Peierls coupling mode, ${ }^{56-69}$ i.e., a fluctuating off-diagonal coupling term in the light-matter interaction $\hat{H}_{\text {int }}$ (Eq. 5), which can modulate the static electronic coupling $\left(V_{\mathrm{DA}}\right)$ between the donor and acceptor state. This, of course, is only valid when the photon frequency approaching to the classical limit $\hbar \omega_{\mathrm{c}} \ll k_{\mathrm{B}} T$.

This Peierls fluctuated electronic coupling for the model in Eq. 5 is expressed as

$$
V_{\mathrm{DA}}\left(Q_{\mathrm{c}}\right)=\left\langle\mathrm{D}\left|\hat{H}_{\mathrm{m}}+\hat{H}_{\mathrm{int}}\right| \mathrm{A}\right\rangle=V_{\mathrm{DA}}+\sqrt{2 \omega_{\mathrm{c}}} g_{\mathrm{c}} Q_{\mathrm{c}} .
$$

The variance $\sigma_{\mathrm{DA}}^{2}$ characterizes the magnitude of the fluctuation around the static value of $\left\langle V_{\mathrm{DA}}\left(Q_{\mathrm{c}}\right)\right\rangle=V_{\mathrm{DA}}$, which is

$$
\sigma_{\mathrm{DA}}^{2}=\left\langle V_{\mathrm{DA}}^{2}\left(Q_{\mathrm{c}}\right)\right\rangle-\left\langle V_{\mathrm{DA}}\left(Q_{\mathrm{c}}\right)\right\rangle^{2}=2 \omega_{\mathrm{c}} g_{\mathrm{c}}^{2}\left\langle Q_{\mathrm{c}}^{2}\right\rangle,
$$

where $\left\langle Q_{\mathrm{c}}^{2}\right\rangle=1 / \beta \omega_{\mathrm{c}}^{2}$ based upon the classical distribution of the photon mode $Q_{\mathrm{c}}$. With the presence of Peierls coupling, the $V_{\mathrm{DA}}$ term in the MT (Eq. 8) needs to be modified as $V_{\mathrm{DA}}\left(Q_{\mathrm{c}}\right)$ (Eq. 10), and the Marcus theory with the Peierls coupling can be expressed as

$$
k_{\mathrm{MT}}^{\mathrm{P}}=\frac{\left\langle V_{\mathrm{DA}}^{2}\left(Q_{\mathrm{c}}\right)\right\rangle}{\hbar} \sqrt{\frac{\pi \beta}{\lambda}} \exp \left[-\beta \frac{(\Delta G+\lambda)^{2}}{4 \lambda}\right],
$$

where the mean square coupling $\left\langle V_{\mathrm{DA}}^{2}\left(Q_{\mathrm{c}}\right)\right\rangle=$ $V_{\mathrm{DA}}^{2}+\sigma_{\mathrm{DA}}^{2}$ includes both static contribution and the fluctuations induced by the photon field. Depending on the relative magnitude of $V_{\mathrm{DA}}^{2}$ and $\sigma_{\mathrm{DA}}^{2}$, the ET rate is controlled by either the averaged electronic coupling square or the variance squared term. ${ }^{61}$

Other Theoretical Approaches. To further assess the accuracy of the ring-polymer quantization of the cavity photon field, we compare it with the following theoretical descriptions of the cavity photon field, where the non-adiabatic dynamics are propagated with Multi-Trajectory Ehrenfest approach (see details in the Supporting Information).

(i) Classical: using a classical distribution of the photon field and Ehrenfest dynamics to propagate the non-adiabatic dynamics.

(ii) Wigner: using the Wigner initial distribution of the photon field ${ }^{25-27}$ and Ehrenfest dynamics to propagate the non-adiabatic dynamics.

(iii) Fock States: using Fock states to repre- 
sent the polariton Hamiltonian $\hat{H}_{\mathrm{pl}}=\hat{H}_{\mathrm{PF}}-\hat{T}_{\mathrm{s}}-$ $\hat{H}_{\mathrm{sb}}$, then propagate the quantum dynamics in the diabatic-Fock basis $\{|\mathrm{D}, n\rangle,|\mathrm{A}, m\rangle\}$.

For all of the above approaches, $10^{4}$ trajectories are used to make sure both convergence and a consistent comparison with the NRPMD calculations. The details of all the above approaches are provided in the Supporting Information.
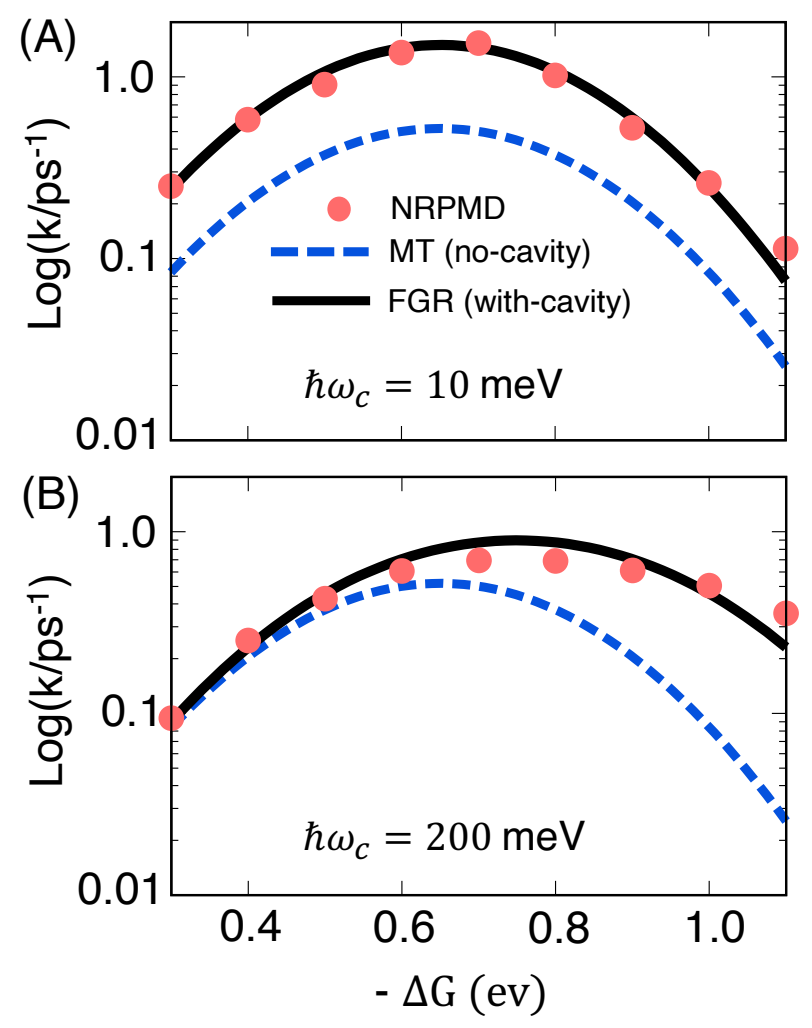

Figure 2: PMET rate constants of molecule-cavity hybrid system over a range of $-\Delta G$, with (A) $\hbar \omega_{\mathrm{c}}=$ $10 \mathrm{meV}$ and $(\mathrm{B}) \hbar \omega_{\mathrm{c}}=200 \mathrm{meV}$. The rate constants are obtained from the NRPMD simulations (red dots), and FGR rate (black solid lines). Marcus theory for the cavity free ET rate constant (blue dashed lines) is presented for comparison.

Results and Discussions. Fig. 2 presents the PMET rate of the molecule-cavity hybrid system over a range of driving force $(-\Delta G)$, with $(\mathrm{A})$ the photon frequency $\hbar \omega_{\mathrm{c}}=10 \mathrm{meV}$ and light-matter coupling strength $\hbar g_{\mathrm{c}}=3 \mathrm{meV}$, as well as (B) $\hbar \omega_{\mathrm{c}}=200 \mathrm{meV}$ and $\hbar g_{\mathrm{c}}=5 \mathrm{meV}$. The NRPMD approach (red dots) with a total of $\mathcal{N}=4$ and $\mathcal{N}=8$ beads are used to generate a converged results for panel A and B, respectively. The PMET rate constants obtained from the NRPMD simulations (red dots) are compared against the FGR rate when the molecule is explicitly coupled to the cavity (black solid lines) and when the molecule is decoupled from the cavity (blue dashed line). For the model used here, the solvent DOF $R_{\mathrm{S}}$ has a low vibrational frequency which does not exhibit any nuclear quantum effects at $T=300 \mathrm{~K}$. As a result, we can see one single turnover of the $\mathrm{ET}$ rate as $-\Delta G$ increases when the molecule is decoupled from the cavity (blue dashed lines in both panels) where the charge transfer occurs via $|\mathrm{D}\rangle \rightarrow|\mathrm{A}\rangle$, and the rate peaks at $-\Delta G=\lambda$, known as the Marcus turnover. ${ }^{51,70}$

With the presence of the cavity, the charge transfer occurs from the photon dressed donor states $|\mathrm{D}, n\rangle$ to the photon dressed acceptor states $|\mathrm{A}, m\rangle$. When the cavity has a low photon frequency $\hbar \omega_{\mathrm{c}}$ $=10 \mathrm{meV}$ (Fig. $2 \mathrm{~A}$ ) such that $\hbar \omega_{\mathrm{c}} \ll k_{\mathrm{B}} T$, one needs to explicitly consider reactive channels for those $n, m \geq 1$. This is because the excited photondressed donor states, $|\mathrm{D}, 1\rangle,|\mathrm{D}, 2\rangle \ldots$, etc, are thermally accessible and as a result, the predominant reactive channel is not only $|\mathrm{D}, 0\rangle \rightarrow|\mathrm{A}, 0\rangle$, but there are also a significant contribution from other high-lying photon-dressed states. As a result, the PMET rate is significantly enhanced throughout all ranges of driving force. Quantizing the radiation mode with a ring-polymer description (through the NRPMD approach) provides quantitatively accurate results compared to the FGR analytical theory.

Fig. 2B presents the PMET rate for the lightmatter hybrid system with a high photonic frequency $\hbar \omega_{\mathrm{c}}=200 \mathrm{meV}$, such that $\hbar \omega_{\mathrm{c}} \gg k_{\mathrm{B}} T$. In this case, the photon frequency is high enough such that under the room temperature, only $|\mathrm{D}, 0\rangle$ has an appreciable amount of thermal population. At a small driving force $-\Delta G<\lambda$, the predominant reactive channel is $|\mathrm{D}, 0\rangle \rightarrow|\mathrm{A}, 0\rangle$, and the channel $|\mathrm{D}, 0\rangle \rightarrow|\mathrm{A}, 1\rangle$ is less favorable due to the large energy difference between these two photondressed states. Hence, the PMET rate constant in this parameter regime is close to the ET rate of the molecule (through the $|\mathrm{D}, 0\rangle \rightarrow|\mathrm{A}, 0\rangle$ reactive channel) without the coupling with the cavity. At a larger driving force, $-\Delta G \geq \lambda$ (Marcus inverted regime), the photon dressed acceptor state $|A, 1\rangle$ is energetically closer to the $|\mathrm{D}, 0\rangle$ state, and hence, the rate constant is higher than the Marcus ET rate in the inverted regime due to this additional channel. Thus, the high frequency radiation mode play a similar role as those high frequency vibrational modes do. ${ }^{54}$ In this sense, the PMET process is akin to the proton-coupled electron transfer reaction (PCET), whereas in PCET, the presence of quantized vibrational levels of the 
transferring proton mediates the effective vibronic couplings as well as the effective state-to-state driving force. ${ }^{71,72}$ Again, ring-polymer description of the photon field provides a quantitatively correct answer compared to the FGR results over almost three orders of magnitude of the rate constants, similar to the success of ring polymer quantization of proton ${ }^{36,73}$ which provides accurate PCET rate constant.
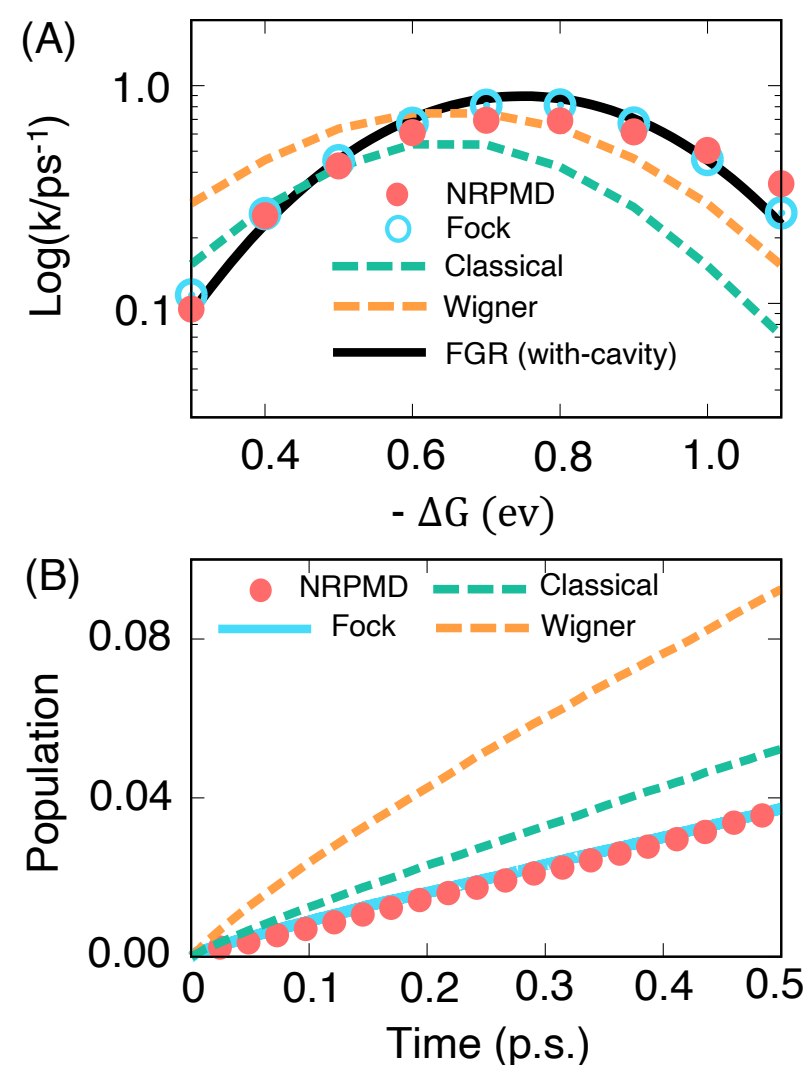

Figure 3: (A) The PMET rate constant of the model system with $\hbar \omega_{\mathrm{c}}=200 \mathrm{meV}$, obtained from different photon quantization approaches, including the ring polymer quantization with NRPMD (red filled circles), the Fock state quantization (cyan open circles), classical description (green dashed line) and Wigner quantization (orange dashed line) of photon mode. (B) The corresponding acceptor state populations.

Fig. 3 presents the detailed comparisons of the PMET rate constants and polariton quantum dynamics obtained from various theoretical treatments of the photon field, including the ring polymer quantization (red dots), Fock state quantization (cyan open circles), Wigner distribution (dashed orange line) and the classical distribution (dashed green line) of the radiation mode.

Fig. 3A presents the PMET rate of the model system with $\hbar \omega_{\mathrm{c}}=200 \mathrm{meV}$ (same model in Fig. 2B) obtained from different theoretical ap- proaches, with FGR rate theory (black solid line) as a benchmark of the quantum result. It can be clearly seen that treating the radiation mode with a classical initial distribution (green dashed line) does not account the quantum effects associated with the high frequency photon modes and thus, fails to predict accurate PMET rate constant throughout the entire range of driving force. Further, in contrast to the previous results of cavity QED, ${ }^{25-28}$ treating the photon mode with initial Wigner distribution (orange dashed line) also fails to provide the quantitative results of the rate constants. The breakdown of the classical Wigner model is likely due to the fact that the classical equation of motion for the photon field in this calculation does not preserve the Wigner distribution, ${ }^{29,31,74}$ a well-known limitation of the classical Wigner model leading to the incorrect flow of the photonic energy to the electronic subsystem. Finally, we also quantize the photon field through the Fock state description (cyan open circles), and this description provides the most accurate results of the PMET rate constant (compared to FGR), due to the explicit quantum mechanical description of the radiation mode as well as all reactive channels. While quantizing the photon field with Fock states provide accurate results, it is limited in terms of how many radiation modes can be explicitly treated. The ring polymer quantization, on the other hand, provides the same level of accuracy while significantly reduce the computational costs by using the extended classical phase space description.

Fig. 3B presents the population dynamics of acceptor state for the same molecule-cavity system presented in Fig. 3A, with a particular driving force $-\Delta G=300 \mathrm{meV}$ to further demonstrate the accuracy of ring-polymer quantization compared to other approaches. We can clearly see that NRPMD (red dots) provides nearly identical population dynamics compared to the Fock state description of the photon field (cyan solid line), which in principle provides the most accurate polariton quantum dynamics (as shown in the rate constant in panel A) . On the other hand, Wigner quantization (orange dashed line) and the classical description (green dashed line) of the photon field fail to provide quantitatively accurate population dynamics.

Fig. 4 compares the PMET results obtained from the fluctuation mediated rate (blue dots) in Eq. 12, the FGR rate (solid black) in Eq. 9, as well as the classical treatment of photon field (green dashed 
line). In Fig. 4A, the model system has the same parameters as the one used in Fig. 2A. At a low photonic frequency, the classical description of the radiation mode provides an accurate result, because the quantum distribution of the photonic DOF is nearly identical with the classical distribution. Further, the fluctuation mediated rate theory provides a quantitative agreement with the FGR rate. In this case, the photon mode can be viewed as a fluctuating Peierls-type of coupling (off-diagonal coupling in the $\{|\mathrm{D}\rangle,|\mathrm{A}\rangle\}$ subspace). The cavity mode fluctuates the value of the electronic coupling (see Eq. 11), and significantly contributes in the rate (see Eq. 12). Under this low frequency regime, the cavity assisted charge transfer mechanism can be purely viewed as the fluctuation of radiation mode that enhance the electronic coupling term. Similar effects have been well understood in charge transfer reactions in protein ${ }^{60,61,64,66,69}$ as well as in singlet fission. ${ }^{75-77}$
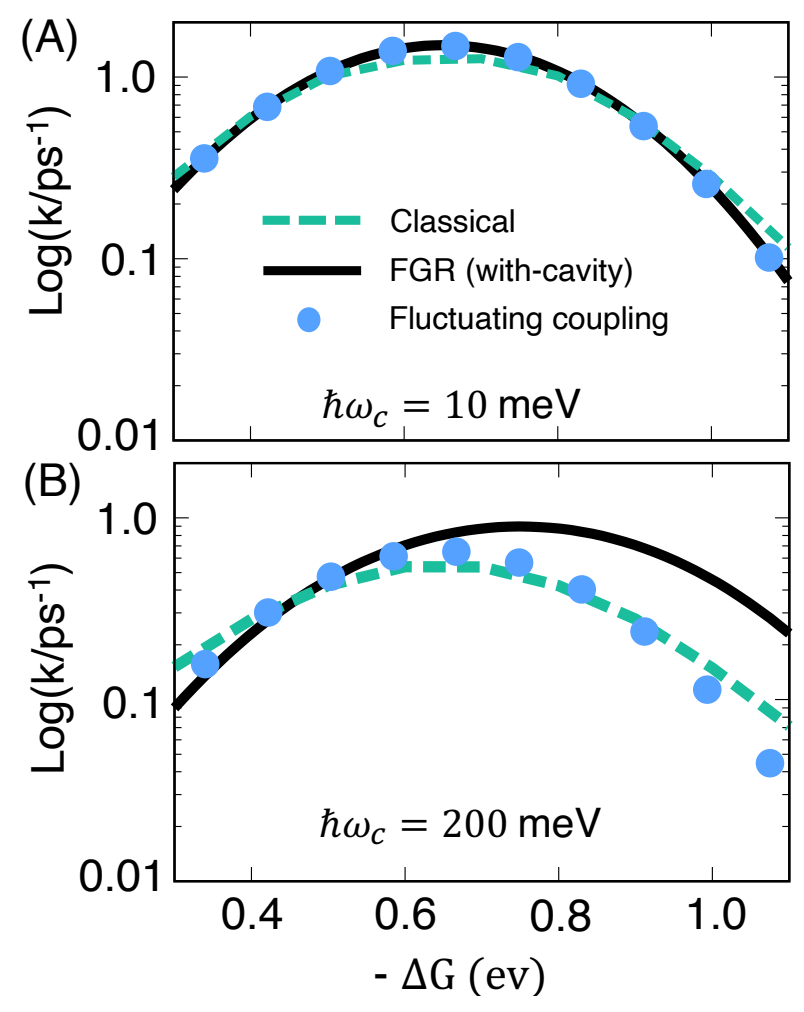

Figure 4: PMET rate constants for molecule-cavity hybrid system over a range of driving force $(-\Delta G)$, with (A) $\hbar \omega_{\mathrm{c}}=10 \mathrm{meV}$ and (B) $\hbar \omega_{\mathrm{c}}=200 \mathrm{meV}$. The rates are obtained with classical description of photon mode (green dash line), FGR rate (black solid lines), and fluctuation mediated rate theory (blue dots).

Fig. 4B presents the same comparison of the model system with a high cavity frequency (same parameters used in Fig. 2B). Due to the high photonic frequency $\hbar \omega_{\mathrm{c}}$, the classical description of photonic DOF is no longer capable to accurately capture the quantum effect, especially the rate enhancement in the Marcus inverted regime. Further, the fluctuation mediated rate theory deviates from the quantum FGR results, but agrees with the classical description of the radiation mode, since both of them use the classical treatment of the radiation mode. On the other hand, both the state-resolved FGR rate and the ring-polymer quantization of the radiation field (Fig. 2B) provide accurate PMET rate constants under the high frequency limit of the cavity mode.

Conclusion. In this letter, to the best of our knowledge, we present the first numerical example of ring-polymer representation to quantize the cavity photon field in polariton chemistry. Using the recently developed non-adiabatic ring-polymer molecular dynamics approach, ${ }^{31,32,37}$ we investigate a charge transfer model coupled to an optical cavity. ${ }^{8,45,48}$ Our numerical results suggest that the ring-polymer quantization of photon field provides an accurate polariton mediated charge transfer rate constants over a broad range of electronic driving force compared to Fermi's Golden Rule. ${ }^{48}$ Our investigation also provides further mechanistic insights into the polariton mediated electron transfer (PMET) reaction. ${ }^{45,48}$ With a high photon frequency $\left(\hbar \omega_{\mathrm{c}}>k_{\mathrm{B}} T\right)$, the cavity radiation mode acts like quantized vibrational DOF, and PMET is analogous to proton-coupled electron transfer reactions. ${ }^{71,72}$ With a low photon frequency $\left(\hbar \omega_{\mathrm{c}}<\right.$ $\left.k_{\mathrm{B}} T\right)$, the cavity mode plays a role of the classical fluctuating Peierls-type coupling, and PMET is analogous to ET reactions in a fluctuating environment such as protein. ${ }^{60,61,64,66,69}$

Compared to the Fock state description of the photon field, the ring-polymer quantization provides the same level of accuracy, and yet offers a computationally convenient framework to describe the polariton quantum effects through the extended phase space description. In contrast to the unfavorable scaling of Fock states, the extended phase space (ring-polymer) description can easily treat multiple quantized modes inside the cavity and scales linearly with these photonic DOF. Compared to the quasi-classical descriptions (such as the classical Wigner model) that do not properly preserve the quantum distribution, ring-polymer preserves the quantum distribution of the photonic 
DOF, resulting in more accurate polaritonic quantum dynamics.

We envision that recently developed statedependent RPMD approaches ${ }^{32,34-37,78-81}$ should be well-suited for the investigation of polariton chemistry $^{27}$ and atomic cavity QED $^{25,26}$ when multiple photonic modes play a crucial role in polariton quantum dynamics. ${ }^{27}$ In fact, because $\left\{\hat{P}_{\mathrm{c}}, \hat{Q}_{\mathrm{c}}\right\}$ in $\hat{H}_{\mathrm{PF}}$ (Eq. 1) can be viewed as an effective "nuclear" DOF that exhibits quantum effects, we conjecture that any approach in theoretical chemistry that can accurately treat nuclear quantization ${ }^{41,43,82-85}$ will have a chance to be applicable to investigate polariton chemistry when explicit quantization of the cavity radiation mode is necessary. This "isomorphism" between the nuclear vibrations and cavity photonic modes could also provide further insights into understanding new reactivities ${ }^{86}$ in polariton chemistry.

\section{Acknowledgment}

This work was supported by the National Science Foundation CAREER Award under the Grant No. CHE1845747, the "Enabling Quantum Leap in Chemistry" program under the Grant No. CHE-1836546, as well as by a Cottrell Scholar award (a program by of Research Corporation for Science Advancement). Computing resources were provided by the Center for Integrated Research Computing at the University of Rochester. S.N.C. appreciates the support from his Arnold Weissberger Fellowship. A.M. appreciates the support from his Elon Huntington Hooker Fellowship. We appreciate valuable discussions with Dr. Duncan Bossion.

\section{References}

(1) Hutchison, J. A.; Schwartz, T.; Genet, C.; Devaux, E.; Ebbesen, T. W. Modifying Chemical Landscapes by Coupling to Vacuum Fields. Angew. Chem. Int. Ed. 2012, 51, 1592-1596.

(2) Thomas, A.; Lethuillier-Karl, L.; Nagarajan, K.; Vergauwe, R. M. A.; George, J.; Chervy, T.; Shalabney, A.; Devaux, E.; Genet, C.; Moran, J.; Ebbesen, T. W. Tilting a Ground-State Reactivity Landscape by Vibrational Strong Coupling. Science 2019, 363, 615-619.

(3) Thomas, A.; Jayachandran, A.; LethuillierKarl, L.; Vergauwe, R. M.; Nagarajan, K.;
Devaux, E.; Genet, C.; Moran, J.; Ebbesen, T. W. Ground State Chemistry Under Vibrational Strong Coupling: Dependence of Thermodynamic Parameters on the Rabi Splitting Energy. Nanophotonics 2020, 9, $249-255$.

(4) Kowalewski, M.; Bennett, K.; Mukamel, S. Cavity Femtochemistry: Manipulating Nonadiabatic Dynamics at Avoided Crossings. J. Phys. Chem. Lett. 2016, 7, 2050-2054.

(5) Galego, J.; Climent, C.; Garcia-Vidal, F. J.; Feist, J. Cavity Casimir-Polder Forces and Their Effects in Ground-State Chemical Reactivity. Phys. Rev. X 2019, 9, 021057.

(6) Schäfer, C.; Ruggenthaler, M.; Rubio, A. Ab Initio Nonrelativistic Quantum Electrodynamics: Bridging Quantum Chemistry and Quantum Optics From Weak to Strong Coupling. Phys. Rev. A 2018, 98, 043801.

(7) Bennett, K.; Kowalewski, M.; Mukamel, S. Novel Photochemistry of Molecular Polaritons in Optical Cavities. Faraday Discuss. 2016, 194, 259-282.

(8) Herrera, F.; Spano, F. C. Cavity-Controlled Chemistry in Molecular Ensembles. Phys. Rev. Lett. 2016, 116, 238301.

(9) Kowalewski, M.; Bennett, K.; Mukamel, S. NonAdiabatic Dynamics of Molecules in Optical Cavities. J. Chem. Phys. 2016, 144, 054309.

(10) Feist, J.; Galego, J.; Garcia-Vidal, F. J. Polaritonic Chemistry with Organic Molecules. ACS Photonics 2018, 5, 205-216.

(11) Triana, J. F.; Peláez, D.; Sanz-Vicario, J. L. Entangled Photonic-Nuclear Molecular Dynamics of $\mathrm{LiF}$ in Quantum Optical Cavities. J. Phys. Chem. A 2018, 122, 2266-2278.

(12) Fregoni, J.; Granucci, G.; Coccia, E.; Persico, M.; Corni, S. Manipulating Azobenzene Photoisomerization Through Strong Light-Molecule Coupling. Nat. Commun. 2018, 9, 4688.

(13) Vendrell, O. Coherent Dynamics in Cavity Femtochemistry: Application of the Multi-Configuration Time-Dependent Hartree Method. Chem. Phys. 2018, 509, 55-65.

(14) Gu, B.; Mukamel, S. Manipulating Nonadiabatic Conical Intersection Dynamics by Optical Cavities. Chem. Sci. 2020, 11, 1290-1298.

(15) Mandal, A.; Huo, P. Investigating New Reactivities Enabled by Polariton Photochemistry. J. Phys. Chem. Lett. 2019, 10, 5519-5529. 
(16) Csehi, A.; Kowalewski, M.; Halász, G. J.; Vibók, Á. Ultrafast Dynamics in the Vicinity of Quantum Light-Induced Conical Intersections. New J. Phys. 2019, 21, 093040.

(17) Szidarovszky, T.; Halász, G. J.; Császár, A. G.; Cederbaum, L. S.; Vibók, A. Conical Intersections Induced by Quantum Light: Field-Dressed Spectra from the Weak to the Ultrastrong Coupling Regimes. J. Phys. Chem. Lett. 2018, 9, 62156223.

(18) Groenhof, G.; Toppari, J. J. Coherent Light Harvesting Through Strong Coupling to Confined Light. J. Phys. Chem. Lett. 2018, 9, 4848-4851.

(19) Groenhof, G.; Climent, C.; Feist, J.; Morozov, D.; Toppari, J. J. Tracking Polariton Relaxation With Multiscale Molecular Dynamics Simulations. J. Phys. Chem. Lett. 2019, 10, 5476-5483.

(20) Rokaj, V.; Welakuh, D. M.; Ruggenthaler, M.; Rubio, A. Light-Matter Interaction in the Longwavelength Limit: No Ground-State Without Dipole Self-Energy. J. Phys. B: At. Mol. Opt. Phys. 2018, 51,034005 .

(21) Flick, J.; Ruggenthaler, M.; Appel, H.; Rubio, A. Atoms and Molecules in Cavities, From Weak to Strong Coupling in Quantum-Electrodynamics (QED) Chemistry. Proc. Natl. Acad. Sci. U. S. A. 2017, 114, 3026-3034.

(22) Mandal, A.; Montillo Vega, S.; Huo, P. Polarized Fock States and the Dynamical Casimir Effect in Molecular Cavity Quantum Electrodynamics. J. Phys. Chem. Lett. 2020,

(23) Miller, W. H. A Classical/Semiclassical Theory for the Interaction of Infrared Radiation With Molecular Systems. J. Chem. Phys 1978, 69, 2188-2195.

(24) Orel, A. E.; Miller, W. H. Infrared Laser Enhancement of Chemical Reactions via Collision Induced Absorption. J. Chem. Phys 1979, 70, 4393-4399.

(25) Hoffmann, N. M.; Schäfer, C.; Rubio, A.; Kelly, A.; Appel, H. Capturing Vacuum Fluctuations and Photon Correlations in Cavity Quantum Electrodynamics With Multitrajectory Ehrenfest Dynamics. Phys. Rev. A 2019, 99, 063819.

(26) Hoffmann, N. M.; Schäfer, C.; Säkkinen, N.; Rubio, A.; Appel, H.; Kelly, A. Benchmarking Semiclassical and Perturbative Methods for Real-Time Simulations of Cavity-Bound Emission and Interference. J. Chem. Phys. 2019, 151, 244113.

(27) Hoffmann, N. M.; Lacombe, L.; Rubio, A.; Maitra, N. T. Effect of Many Modes on SelfPolarization and Photochemical Suppression in Cavities. J. Chem. Phys. 2020, 153, 104103.
(28) Li, T. E.; Chen, H.-T.; Nitzan, A.; Subotnik, J. E. Quasiclassical Modeling of Cavity Quantum Electrodynamics. Phys. Rev. A 2020, 101, 033831.

(29) Habershon, S.; Manolopoulos, D. E. Zero Point Energy Leakage in Condensed Phase Dynamics: An Assessment of Quantum Simulation Methods for Liquid Water. J. Chem. Phys. 2009, 131, 244518.

(30) Muller, U.; Stock, G. Flow of Zero-Point Energy and Exploration of Phase Space in Classical Simulations of Quantum Relaxation Dynamics. II. Application to Nonadiabatic Processes. J. Chem. Phys. 1999, 111, 77.

(31) Chowdhury, S. N.; Huo, P. State Dependent Ring Polymer Molecular Dynamics for Investigating Excited Nonadiabatic Dynamics. J. Chem. Phys. 2019, 150, 244102.

(32) Richardson, J. O.; Thoss, M. Nonadiabatic Ring Polymer Molecular Dynamics. J. Chem. Phys. 2013, 139, 031102.

(33) Hele, T. J. H.; Ananth, N. Deriving the Exact Nonadiabatic Quantum Propagator in the Mapping Variable Representation. Faraday Discuss. 2016, 195, 269.

(34) Ananth, N. Mapping Variable Ring Polymer Molecular Dynamics: A Path-Integral Based Method for Nonadiabatic Processes. J. Chem. Phys. 2013, 139, 124102.

(35) J.R.Duke,; Ananth, N. Simulating Excited State Dynamics in Systems With Multiple Avoided Crossings Using Mapping Variable Ring Polymer Molecular Dynamics. J. Phys. Chem. Lett. 2015, 06,4219 .

(36) Pierre, S.; Duke, J. R.; Hele, T. J. H.; Ananth, N. A Mapping Variable Ring Polymer Molecular Dynamics Study of Condensed Phase ProtonCoupled Electron Transfer. J. Chem. Phys. 2017, $147,234103$.

(37) Richardson, J. O.; Meyer, P.; Pleinert, M.-O.; Thoss, M. An Analysis of Nonadiabatic RingPolymer Molecular Dynamics and Its Application to Vibronic Spectra. Chem. Phys. 2017, 482, 124 -134 .

(38) Berne, B. J.; Thirumalai, D. On the Simulation of Quantum Systems: Path Integral Methods. Annu. Rev. Phys. Chem. 1986, 37, 401.

(39) Ceperley, D. M. Path Integrals in the Theory of Condensed Helium. Rev. Mod. Phys. 1995, 67, 279. 
(40) Chandler, D.; Wolynes, P. G. Exploiting the Isomorphism Between Quantum Theory and Classical Statistical Mechanics of Polyatomic Fluids. J. Chem. Phys. 1981, 74, 4078.

(41) Cao, J.; Voth, G. A. The Formulation of Quantum Statistical Mechanics Based on the Feynman Path Centroid Density. II. Dynamical Properties. J. Chem. Phys. 1994, 100, 5106-5117.

(42) Craig, I. R.; Manolopoulos, D. E. Quantum Statistics and Classical Mechanics: Real Time Correlation Functions From Ring Polymer Molecular Dynamics. J. Chem. Phys. 2004, 121, 3368.

(43) Habershon, S.; Manolopoulos, D. E.; Markland, T. E.; Miller, T. F. Ring Polymer Molecular Dynamics: Quantum Effects in Chemical Dynamics From Classical Trajectories in an Extended Phase Space. Annu. Rev. Phys. Chem. 2013, 64, 124105 .

(44) Cohen-Tannoudji, C.; Dupont-Roc, J.; Grynberg, G. Photons and Atoms: Introduction to Quantum Electrodynamics. John Wiley 83 Sons, Inc.: Hoboken, U.S.A. 1989,

(45) Mandal, A.; Krauss, T. D.; Huo, P. PolaritonMediated Electron Transfer via Cavity Quantum Electrodynamics. J. Phys. Chem. B 2020, 124, 6321-6340.

(46) Meyer, H. D.; Miller, W. H. A Classical Analog for Electronic Degrees of Freedom in Nonadiabatic Collision Processes. J. Chem. Phys 1979, 70, 3214 .

(47) Stock, G.; Thoss, M. Semiclassical Description of Nonadiabatic Quantum Dynamics. Phys. Rev. Lett. 1997, 78, 578.

(48) Semenov, A.; Nitzan, A. Electron Transfer in Confined Electromagnetic Fields. J. Chem. Phys. 2019, 150, 174122.

(49) Jain, A.; Subotnik, J. E. Does Nonadiabatic Transition State Theory Make Sense Without Decoherence? J. Phys. Chem. Lett. 2015, 6, 4809-4814.

(50) Jain, A.; Subotnik, J. E. Surface Hopping, Transition State Theory, and Decoherence. II. J. Chem. Phys. 2015, 143, 134107.

(51) Marcus, R. A. On the Theory of OxidationReduction Reactions Involving Electron Transfer. I. J. Chem. Phys. 1956, 24, 966-978.

(52) Campos-Gonzalez-Angulo, J. A.; Ribeiro, R. F.; Yuen-Zhou, J. Resonant Catalysis of ThermallyActivated Chemical Reactions With Vibrational Polaritons. Nat. Commun. 2019, 10, 4685.
(53) Nitzan, A.; Jortner, J.; Rentzepis, P. M.; Porter, G. Intermediate Level Structure in Highly Excited Electronic States of Large Molecules. Proc. R. Soc. London, Ser. A 1972, 327, 367-391.

(54) Ulstrup, J.; Jortner, J. The Effect of Intramolecular Quantum Modes on Free Energy Relationships for Electron Transfer Reactions. J. Chem. Phys. 1975, 63, 4358-4368.

(55) Efrima, S.; Bixon, M. On the Role of Vibrational Excitation in Electron Transfer Reactions With Large Negative Free Energies. Chem. Phys. Lett. 1974, 25, 34-37.

(56) Davis, W. B.; Ratner, M. A.; Wasielewski, M. R. Conformational Gating of Long Distance Electron Transfer through Wire-like Bridges in DonorBridge-Acceptor Molecules. J. Am. Chem. Soc. 2001, 123, 7877-7886.

(57) Beratan, D. N.; Onuchic, J. N.; Hopfield, J. J. Electron Tunneling Through Covalent and Noncovalent Pathways in Proteins. J. Chem. Phys. 1987, 86, 4488-4498.

(58) Beratan, D. N.; Onuchic, J. N. Electron Tunneling Pathways in Proteins: Influences on the Transfer Rate. Photosynth. Res. 1989, 22, 173-186.

(59) Onuchic, J. N.; Beratan, D. N. A Predictive Theoretical Model for Electron Tunneling Pathways in Proteins. J. Chem. Phys. 1990, 92, 722-733.

(60) Troisi, A.; Nitzan, A.; Ratner, M. A. A Rate Constant Expression for Charge Transfer Through Fluctuating Bridges. J. Chem. Phys. 2003, 119, $5782-5788$.

(61) Skourtis, S. S.; Waldeck, D. H.; Beratan, D. N. Fluctuations in Biological and Bioinspired Electron-Transfer Reactions. Annu. Rev. Phys. Chem. 2010, 61, 461-485.

(62) Troisi, A.; Nitzan, A.; Ratner, M. A. A Rate Constant Expression for Charge Transfer Through Fluctuating Bridges. J. Chem. Phys. 2003, 119, $5782-5788$.

(63) Goychuk, I. A.; Petrov, E. G.; May, V. BridgeAssisted Electron Transfer Driven by Dichotomically Fluctuating Tunneling Coupling. J. Chem. Phys. 1995, 103, 4937-4944.

(64) Beratan, D. N.; Skourtis, S.; Balabin, I. A.; Balaeff, A.; Keinan, S.; Venkatramani, R.; Xiao, D. Steering Electrons on Moving Pathways. Acc. Chem. Res. 2009, 42, 1669-1678. 
(65) Prytkova, T. R.; Kurnikov, I. V.; Beratan, D. N. Coupling Coherence Distinguishes Structure Sensitivity in Protein Electron Transfer. Science 2007, 315, 622-625.

(66) Antony, J.; Medvedev, D. M.; Stuchebrukhov, A. A. Theoretical Study of Electron Transfer Between the Photolyase Catalytic Cofactor FADH- and DNA Thymine Dimer. J. Am. Chem. Soc. 2000, 122, 1057-1065.

(67) Medvedev, E. S.; Stuchebrukhov, A. A. Inelastic Tunneling in Long-Distance Biological Electron Transfer Reactions. J. Chem. Phys. 1997, 10\%, 3821-3831.

(68) Daizadeh, I.; Medvedev, E. S.; Stuchebrukhov, A. A. Effect of Protein Dynamics on Biological Electron Transfer. Proc. Natl. Acad. Sci. USA 1997, 94, 3703-3708.

(69) Ma, Z.; Lin, Z.; Lawrence, C. M.; Rubtsov, I. V.; Antoniou, P.; Skourtis, S. S.; Zhang, P.; Beratan, D. N. How Can Infra-Red Excitation Both Accelarate and Slow Charge Transfer in the Same Molecule? Chemical Science 2018, 9, 6395.

(70) Marcus, R. A.; Sutin, N. Electron Transfers in Chemistry and Biology. Biochim. Biophys. Acta, Biophys. Incl. Photsynth. 1985, 811, 265-322.

(71) Hammes-Schiffer, S.; Stuchebrukhov, A. A. Theory of Coupled Electron and Proton Transfer Reactions. Chem. Rev. 2010, 110, 6939-6960.

(72) Hammes-Schiffer, S. Proton-Coupled Electron Transfer: Classification Scheme and Guide to Theoretical Methods. Energy Environ. Sci. 2012, 5, 7696-7703.

(73) Kretchmer, J.; Miller, T. Direct simulation of proton-coupled electron transfer across multiple regimes. J. Chem. Phys. 2013, 138, 134109.

(74) Liu, J.; Miller, W. H. An Approach for Generating Trajectory-Based Dynamics Which Conserves the Canonical Distribution in the Phase Space Formulation of Quantum Mechanics. I. Theories. J. Chem. Phys. 2011, 134, 104101.

(75) Renaud, N.; Grozema, F. C. Intermolecular Vibrational Modes Speed Up Singlet Fission in Perylenediimide Crystals. J. Phys. Chem. Lett. 2015, 6, 360-365.

(76) Arago, J.; Troisi, A. Dynamics of the Excitonic Coupling in Organic Crystals. Phys. Rev. Lett. 2015, 8, 026402.
(77) Castellanos, M.; Huo, P. Enhancing SingletFission Dynamics by Suppressing Destructive Interference between Charge-Transfer Pathways. J. Phys. Chem. Lett. 2017, 8, 2480-2488.

(78) Chowdhury, S. N.; Huo, P. Coherent State Mapping Ring Polymer Molecular Dynamics for NonAdiabatic Quantum Propagations. J. Chem. Phys 2017, 147, 214109.

(79) Menzeleev, A. R.; Bell, F.; Miller, T. F. Kinetically Constrained Ring-Polymer Molecular Dynamics for Non-Adiabatic Chemical Reactions. $J$. Chem. Phys. 2014, 140, 064103.

(80) Kretchmer, J.; Miller, T. F. Tipping the Balance Between Concerted Versus Sequential ProtonCoupled Electron Transfer. Inorg. Chem. 2016, 55, 1022-1031.

(81) Kretchmer, J.; Boekelheide, N.; Warren, J. F.; Winkler, J. R.; Gray, H. B.; Miller, T. F. Fluctuating Hydrogen-Bond Networks Govern Anomalous Electron Transfer Kinetics in a Blue Copper Protein. Proc. Natl. Acad. Sci. USA 2018, 115, 6129-6134.

(82) Jang,; Voth, G. A Derivation of Centroid Molecular Dynamics and Other Approximate Time Evolution Methods for Path Integral Centroid Variables. J. Chem. Phys. 1999, 111, 2371.

(83) Liao, J.-L.; Voth, G. A. A Centroid Molecular Dynamics Approach for Nonadiabatic Dynamical Processes in Condensed Phases: the Spin-Boson Case. J. Phys. Chem. B. 2002, 106, 8449-8455.

(84) Curchod, B. F. E.; Martinez, T. J. Ab initio Nonadiabatic Quantum Molecular Dynamics. Chem. Rev. 2018, 118, 3305-3336.

(85) Richings, G.; Polyak, I.; Spinlove, K.; Worth, G.; Burghardt, I.; Lasorne, B. Quantum Dynamics Simulations Using Gaussian Wavepackets: The vMCG Method. Int. Rev. Phys. Chem. 2015, 34, 269-308.

(86) Ebbesen, T. W. Hybrid Light-Matter States in a Molecular and Material Science Perspective. Acc. Chem. Res. 2016, 49, 2403-2412. 